\title{
Excellence académique, joutes oratoires et pragmatisme pédagogique à Singapour
}

Academic excellence, oratorical spars and pedagogical pragmatism in Singapore Excelencia académica, justas oratorias y pragmatismo pedagógico en Singapur

\section{Corinne Rousset}

\section{OpenEdition}

\section{Journals}

Édition électronique

URL : https://journals.openedition.org/ries/6131

DOI : $10.4000 /$ ries.6131

ISSN : 2261-4265

\section{Éditeur}

France Education international

\section{Édition imprimée}

Date de publication : 30 avril 2018

Pagination : 59-69

ISBN : 978-2-85420-618-0

ISSN : $1254-4590$

\section{Référence électronique}

Corinne Rousset, «Excellence académique, joutes oratoires et pragmatisme pédagogique à

Singapour ", Revue internationale d'éducation de Sèvres [En ligne], 77 | avril 2018, mis en ligne le 30 avri 2020, consulté le 25 juin 2021. URL : http://journals.openedition.org/ries/6131 ; DOI : https://doi.org/ $10.4000 /$ ries. 6131

(c) Tous droits réservés 


\section{Excellence académique, joutes oratoires et pragmatisme pédagogique à Singapour}

\section{Corinne Rousset \\ Université de Tours}

Depuis quelques années, Singapour caracole en tête des enquêtes Pisa (De Ketele, 2014). Les élèves singapouriens se distinguent dans trois domaines de compétence : sciences, mathématiques et lecture. Ces résultats hors du commun sont le reflet d'un système extrêmement compétitif, focalisé sur l'efficacité et la performance. Les élèves et leurs parents sont motivés par la réussite académique, tremplin vers une réussite économique qui confine à l'obsession.

Pour comprendre cette réussite, il nous faut retourner brièvement à l'histoire du pays et à ses origines. Dès 1965, date de l'indépendance fragile et du début de la gouvernance de Lee Kuan Yew, Singapour dresse ce constat réaliste : le pays ne dispose d'aucune ressource naturelle. Qu'à cela ne tienne, il se dotera de travailleurs hautement qualifiés. Le chef d'État met en place sa vision ambitieuse et conçoit un système d'éducation élitiste, reposant sur la méritocratie, où les mathématiques et les sciences sont élevées au rang de priorité nationale, avec l'anglais comme langue officielle et langue d'enseignement, choix qui facilitera grandement l'accès au statut de place financière internationale qu'acquiert rapidement le pays. Cinquante ans plus tard, le pari est réussi. Plusieurs ethnies cohabitent dans ce pays multiconfessionnel : Chinois, Indiens, Malais et un grand nombre d'expatriés séduits par une fiscalité et des salaires attractifs. En outre, beaucoup d'Asiatiques ont élu aujourd'hui la cité-État comme lieu d'éducation de leurs enfants, en raison de la sécurité qu'elle offre par rapport aux pays de la région. De nombreux élèves étrangers étudient, seuls ou avec leurs parents, et parfois dès l'âge de l'entrée au collège, dans les écoles locales ou internationales et autres internats de Singapour, qui se targue désormais d'être la plateforme asiatique de l'éducation. Ainsi, en plus d'un réseau de 365 écoles publiques locales accueillant 450000 élèves, Singapour dispose d'un réseau de 34 écoles internationales accueillant environ 50000 élèves, fondées par des institutions privées. L'anglais étant langue d'enseignement, les programmes scolaires sélectionnés sont ceux de Cambridge et les élèves passent l'International General Certificate of Secondary Education (IGCSE) ou le O-level en fin de secondaire 
inférieur et le A-level en fin de secondaire supérieur. Dans les écoles internationales, le programme dispensé est celui du Baccalauréat international, reconnu dans les universités du monde entier.

Enfin, Singapour étant le pays concentrant le plus grand nombre de milliardaires au monde (et au troisième rang mondial des richesses par habitant), les enfants accèdent très aisément à la technologie. Dans ce petit pays, où, malgré une appétence certaine pour le sport, les activités en extérieur peuvent parfois être limitées en raison du climat équatorial, les jeunes passent en moyenne cinq heures par jour sur Internet et sont donc très tôt confrontés à de multiples informations glanées sur les médias et réseaux en ligne, comme tous les jeunes occidentaux. Dans ce contexte plurinational et multiconfessionnel, les enseignants en charge de la formation des élèves sont confrontés à un triple défi : assurer un enseignement de qualité permettant d'atteindre les objectifs d'excellence fixés par le ministère de l'éducation, tout en intégrant des profils d'apprenants variés, issus de nationalités, cultures et religions diverses et présentant des connaissances et des systèmes de valeurs potentiellement différents mais cependant exposés à une communication globalisée, et par conséquent à des informations peu identifiables issues des réseaux sociaux et diffusées en masse.

\section{L'OBSESSION DE RÉUSSITE DES ÉLÈVES : UN FREIN À LA CONTESTATION}

Motivés par la réussite, les élèves ont le souci constant, voire obsessionnel, d'obtenir les meilleures notes et classements possibles. Le système étant fondé sur la méritocratie, seuls 30 \% des élèves accèdent à l'enseignement supérieur. Les parents poussent donc les enfants dès leur plus jeune âge, exerçant une pression parfois insoutenable sur les notes. $70 \%$ des élèves fréquentent de coûteux centres d'étude et de soutien après l'école, où ils reçoivent l'aide de tuteurs spécialisés pour revoir le programme ou bénéficier d'une aide aux devoirs.

Cette situation particulière fait de l'enseignant le transmetteur d'un savoir et d'une connaissance indispensables aux élèves, si ces derniers visent la réussite scolaire tant souhaitée. Par conséquent, les contenus des programmes font rarement l'objet de contestations, puisqu'ils qu'ils constituent un passage obligé dans le parcours du combattant de l'élève singapourien.

Cette ambition s'accompagne d'une disposition et d'un état d'esprit très spécifiques aux valeurs asiatiques et singapouriennes en particulier. Sous l'influence de Lee Kuan Yew, l'obéissance et le respect des institutions sont les valeurs sociales mises en œuvre avec détermination dès la création de l'État. Elles sont très présentes dans toutes les dimensions de la vie quotidienne et leur apprentissage commence dès l'école primaire. Chaque jour, tous les élèves chantent l'hymne national et récitent le serment d'allégeance à la patrie. Les professeurs sont les garants de cette adhésion nationale et, ainsi, le rapport 
de l'élève à l'enseignant bascule tacitement en un rapport de respect, voire de soumission. La quasi sacralisation des connaissances académiques - et en particulier des matières scientifiques - rend incongrue toute confrontation sur le savoir détenu par l'enseignant et sur les programmes enseignés.

Comme nous l'avons dit, Singapour est un pays international et multiconfessionnel. Beaucoup d'élèves et de professeurs provenant de pays, traditions et cultures, religions et systèmes juridiques différents s'y côtoient harmonieusement. Ces caractéristiques nationales et culturelles ne semblent pas constituer un problème insurmontable dans la façon dont les élèves abordent le contenu des programmes. En effet, le système attire, grâce à son élitisme. Ce puissant moteur d'assimilation semble prendre le pas sur les différences culturelles, aussi importantes soient-elles, et décourager davantage les potentielles résistances, confrontations et oppositions de nature épistémologique des élèves à l'égard de la connaissance délivrée par le corps enseignant (Tan, 2017).

\section{L'ENSEIGNEMENT DE LA BIOLOGIE AU CEUR DES MATIÈRES SCIENTIFIQUES ${ }^{1}$}

Les enseignants de biologie sont rarement confrontés à des assertions incompatibles avec les théories au programme. Leur approche repose systématiquement sur le rationalisme, c'est-à-dire sur l'analyse des évidences et sur le recours à la méthode scientifique, peu propices à la contestation en classe. L'étude de la génétique, de l'épigénétique et de l'ADN sont des composantes importantes des programmes de biologie et leur enseignement repose sur des données vérifiables, peu contestables et peu contestées.

Un grand nombre d'écoles de tradition religieuse chrétienne dispensent des enseignements religieux, qui n'interfèrent cependant pas avec la théorie de l'évolution, qui reste incontestée dans les enseignements dispensés à Singapour. S'il a pu arriver que des personnalités religieuses évoquent le créationnisme ou contestent la théorie du Big Bang dans certaines églises, les professeurs ne se trouvent pas en situation de devoir justifier les théories scientifiques ou de défendre la théorie de l'évolution car les élèves à Singapour se satisfont tout à fait des explications scientifiques incluses dans les programmes d'enseignement,

1. Cet article a été rédigé sur la base d'un ensemble de réponses à un panel de questions posées à des enseignants du secondaire en biologie, histoire, langues, art et théorie de la connaissance, intervenant à Singapour dans établissements locaux et internationaux, publics et privés, ainsi que dans deux universités nationales. Les questions portaient de manière ciblée et pratique sur les situations (même anecdotiques) rencontrées par ces professeurs lors de l'exposition à des vérités contradictoires, théories douteuses et autres théories du complot, et sur les éventuelles stratégies utilisées par ces mêmes enseignants, en cours, pour accompagner les élèves ou les étudiants dans cette réflexion sur la vérité. Il a été également demandé aux enseignants ayant eu une expérience professionnelle dans d'autres pays de montrer les spécificités de l'enseignement à Singapour par rapport à ces problématiques. 
qu'ils placent au panthéon de la hiérarchie de la connaissance. Le ministère de l'éducation a d'ailleurs très directement clarifié ce point et confirmé que la théorie de l'évolution répondait aux objectifs d'évaluation critique des connaissances en science fondamentale. Ironiquement, ce sont les enseignants de biologie qui se trouvent parfois confrontés à leurs propres contradictions. Ainsi, un certain nombre d'entre eux se présentant volontiers comme croyants, notamment dans les écoles affichant une appartenance religieuse, ont résolu leurs dilemmes (la vie est-elle une création divine ou la conséquence d'une série de hasards biologiques ?) en utilisant les mathématiques et en invitant les élèves à envisager la probabilité que tous les éléments en place afin que la vie puisse exister soient le seul fait du hasard ou d'une volonté supérieure, laissant ainsi aux élèves le soin de réfléchir à la question par eux-mêmes, alors qu'ils ne se l'étaient sans doute pas posée spontanément. Ces anecdotes confirment que la capacité des enseignants à appréhender ces thèmes controversés dépend largement de leur propre perception de ces sujets (Seow et Ho, 2015).

Ces questionnements restent cependant minoritaires. D'une manière générale, les élèves opèrent une distinction relativement étanche entre les concepts présentés par les institutions religieuses (qui représentent une importante partie de la vie sociale à Singapour) et les contenus académiques. L'injonction de réussite aux examens, qui se traduit par l'assimilation la plus fidèle et la plus fiable possible des programmes scientifiques (y compris des théories au programme) explique largement ce phénomène.

\section{UNE APPROCHE RATIONALISTE ET PRAGMATIQUE DES DÉBATS DU MOMENT}

La question du réchauffement climatique semble un peu plus propice non pas à la contestation mais à l'exercice du débat comme stratégie d'investissement des connaissances et d'analyse critique en sciences. La plupart des professeurs accueillent ou proposent ce thème comme une opportunité pédagogique et abordent d'eux-mêmes la question de la validité des discours contestant la responsabilité humaine dans le réchauffement climatique. Ils se montrent prompts à ouvrir le dialogue et proposent régulièrement aux élèves de faire état de leurs connaissances ou des théories auxquelles ils ont pu être exposés dans les médias. Les élèves sont invités à scruter la source des documents et à décrypter les écoles de pensées à l'œuvre derrière certaines assertions, à vérifier les données présentées comme scientifiques, à analyser les parties prenantes politiques ou idéologiques du débat. Cet exercice possède un double avantage : celui de vérifier les modalités d'évaluation de toute assertion scientifique mais aussi de situer la discipline de la biologie dans la vie et l'actualité du monde. Pour autant, ce sujet ne passionne pas autant à Singapour qu'ailleurs. Le réchauffement climatique, même s'il y est parfaitement compris et plutôt 
incontesté, n'est pas vécu comme une menace majeure et en tous cas ne saurait inciter les jeunes qui étudient à Singapour à modifier un comportement consumériste qu'ils adoptent volontiers. ${ }^{2}$

Une autre particularité culturelle observable en cours de biologie est l'attitude face aux enseignements touchant à la sexualité et à la reproduction. Les cultures asiatiques étant traditionnellement réservées et pudiques, ces enseignements sont particulièrement appréciés des élèves car ils leur offrent la possibilité d'aborder des sujets qui restent tabous dans les cercles familiaux. La classe, lorsque ces parties du programme sont abordées (qu'il s'agisse des maladies sexuellement transmissibles, de la reproduction ou des technologies d'aide à la reproduction), devient lieu de transmission de connaissances non plus uniquement scientifiques mais fondamentales. En cela, l'école remplace bien souvent l'enseignement traditionnellement dévolu aux parents et à la famille dans d'autres pays ou cultures. Ainsi, différentes thèses développées dans des vidéos vues sur les réseaux sociaux ou simplement entendues et relayées par les élèves sont régulièrement mentionnées en classe par les élèves, telle que la prétendue contamination au VIH par la salive. Pour traiter ces cas, quand ils se présentent spontanément, les professeurs adoptent une approche pragmatique et rationaliste, n'hésitant pas à visionner les vidéos avec les élèves pendant le cours, afin de relever les données et les arguments présentés, qui sont ensuite passés au crible de la méthode scientifique et comparés aux connaissances universellement reconnues dans ce domaine. Ainsi, les théories divergentes et autres assertions ne sont jamais balayées aveuglément ou directement corrigées par les enseignants : la pédagogie repose sur l'étude des données du cas, sur l'invitation faite aux élèves à s'emparer eux-mêmes des outils méthodologiques acquis, afin de valider ou non la validité des thèses en question. L'approche se veut donc rationaliste et pragmatique.

\section{LA PRISE EN COMPTE DES QUESTIONS D'ÉTHIQUE EN CLASSE}

Quelques problématiques d'ordre éthique donnent régulièrement lieu à des débats et questionnements en classe, lorsqu'elles touchent à la vie. Elles apparaissent notamment lorsque sont abordés des sujets tels que les cellules souches, les procédures de procréation in vitro ou de gestation pour autrui, le problème légal des mères porteuses et des droits de l'enfant, surtout s'il est né dans des dispositions contraires à la loi singapourienne.

2. Classé au $7^{\mathrm{e}}$ rang des pollueurs au titre de son empreinte écologique, Singapour se caractérise par son profil d'hyperconsommation et a, en ceci, rejoint le modèle des pays à fort revenu, selon un rapport de WWF (2014): [https://goo.gl/89Eg4A]. Singapour conteste les méthodes de classement utilisées par WWF. Notons cependant que le pays a signé les accords de Paris en 2017, taxe désormais les émetteurs de carbone et s'est engagé dans une politique environnementale volontariste. 
Plus rarement, ce sont les élèves qui soulèvent eux-mêmes certaines problématiques, comme le déterminisme biologique dans l'homosexualité. Tous ces sujets constituent des occasions typiques, pour certains discours conservateurs et politiques (au niveau national, institutionnel ou parental) d'entrer en conflit avec les théories scientifiques. Quelle que soit la classe dans laquelle ces thèmes sont abordés (scientifique ou humaniste, comme la théorie de la connaissance) ${ }^{3}$, les élèves sont rarement indifférents. Par exemple, les enseignants sont presque toujours interpellés sur la question de la destruction des embryons surnuméraires.

Les approches et attitudes pédagogiques passent par une prise en compte des opinions et ressentis des élèves, qui souvent mettent en avant leurs propres systèmes de lois, valeurs et traditions culturelles ou religieuses, lesquelles ne sont pas toujours compatibles entre elles puisque dans ces classes cohabitent la plupart du temps différentes cultures. On aborde ici la difficulté bien connue, qui soustend tout enseignement dans un contexte de culture "hybride", comme à Singapour, où la défense d'une théorie ou d'un dogme pourrait s'avérer intenable, alors que nous vivons paradoxalement dans une ère de globalisation, dont on pourrait attendre à ce qu'elle fournisse un prêt à penser standardisé. Pour mener à bien sa mission d'enseignement, l'enseignant endosse alors un rôle de facilitateur de l'expression de ces divergences, et recourt aux stratégies qui lui correspondent le mieux, en fonction de sa propre sensibilité et de son expérience.

Des jeux de rôle, dans lesquels il s'agit de défendre une thèse que l'on ne soutient pas, peuvent être organisés afin de permettre aux élèves de mieux comprendre les positions d'autrui et d'en comprendre les origines. Ces exercices ne constituent cependant qu'une partie modeste des activités proposées en classe. Les évaluations ne portent que sur les connaissances qu'il s'agit de maîtriser en vue des examens. Au regret clairement exprimé de certains professeurs, les élèves, notamment au cours des enquêtes de satisfaction, manifestent continuellement leur désir de se focaliser sur l'apprentissage des parties du programme sanctionnées aux examens plutôt que sur les activités purement épistémologiques et de réflexion.

\section{L'ART DU DÉBAT POUR DÉCONSTRUIRE LES THÉORIES FALLACIEUSES}

Il existe cependant un aspect propre à l'enseignement à Singapour suffisamment paradoxal pour être relevé, et qui reflète assez bien la culture locale : le débat. Singapour a gagné déjà par deux fois le championnat d'écoles mondial depuis ces vingt dernières années. Ces résultats exceptionnels sont le résultat d'une culture nationale tout à fait particulière : chaque élève dans chaque école

3. La théorie de la connaissance est un cours obligatoire sur deux ans (les deux dernières années du secondaire) dans le programme du Baccalauréat international. Ce cours de réflexion critique et d'épistémologie porte sur la façon dont sont acquises les connaissances en sciences naturelles, sciences sociales, arts, religion, histoire, mathématiques et dans les systèmes de connaissance indigènes. Pour en savoir plus : [https://goo.gl/SsRMa3] 
dispose de la possibilité de s'inscrire à une classe optionnelle et gratuite d'initiation au débat, menée par un coach professionnel, formé aux techniques de débat adoptées dans les compétitions internationales, et ce dès les petites classes.

Rapidement rompus à cet exercice pourtant difficile, les élèves sont donc accoutumés à débattre dans les règles de l'art. Cela peut prendre la forme d'une présentation préparée, d'un exercice de rhétorique chronométré, où l'on forme des équipes et où chaque camp prend en charge une idée ou une thèse que les élèves n'épouseraient pas spontanément et où chaque argument correctement justifié vaut un point. Dans la plupart des cas, ces exercices aident les élèves à comprendre les enjeux que soulèvent la gouvernance et la législation sur des sujets éthiques spécifiques, lorsque les règles religieuses ou les traditions culturelles contredisent le droit civil, le paradoxe entre droits de l'homme et utilitarisme, la géométrie variable du mot "vérité ", la complexité d'établir la validité de toute théorie, etc. De fait, l'exercice les expose à des sujets contemporains, développant ainsi leur sens critique et leur conscience éthique et citoyenne. Si ces techniques sont très régulièrement utilisées en classe et appréciées des élèves, seuls les meilleurs élèves réussirent à concourir dans ces joutes oratoires au niveau national. Pour autant, ces compétitions scolaires publiques, qui constituent des moments prisés de la vie des écoles, ont d'ailleurs lieu lors d'événements auxquels assistent souvent tous les élèves de l'établissement. Apprendre à identifier les manipulations d'arguments, à déjouer les sophismes et à maîtriser les techniques de déconstruction de théories fallacieuses reste donc un exercice relativement familier pour tout élève fréquentant l'école publique ou privée à Singapour. L'art de l'éloquence et du débat constitue un sport national, qui rappelle l'époque grecque. Mais, plutôt que d'une passion pour la quête de vérité, il s'agit surtout d'une énième démonstration de l'appétit singapourien pour la compétition, même si certains champions de l'éloquence deviennent indéniablement des individus intellectuellement plus curieux.

\section{APPRENDRE À REPÉRER LES TECHNIQUES DE MANIPULATION EN COURS D'HISTOIRE ET DE THÉORIE DE LA CONNAISSANCE}

Malgré toutes ces aptitudes remarquables, le principal problème rencontré par les enseignants est la frustration face à des élèves essentiellement motivés par l'obtention des meilleures notes possibles, au dépend d'une pensée critique plus personnelle.

Dans les facultés d'histoire et de théorie de la connaissance, les enseignants enseignent la capacité à analyser les sources d'information, ainsi que le discernement face à l'information présentée. La plupart des élèves sont donc formés à ces techniques mais peu s'y intéressent sincèrement, même si une 
minorité d'entre eux a déjà recours à des sources fiables, comme le site de la BBC ou du New York Times. Cette pédagogie est relativement simple à mettre en œuvre à tous les âges; les enseignants confrontent les élèves à toutes sortes de matériaux de propagande (presse écrite, textes numériques, discours, photos, etc.) et les forment à analyser ces sources, en mettant l'accent sur l'identification des logiques et raisonnements fallacieux, les techniques de manipulation du langage, la recontextualisation de visuels photographiques et le décryptage de l'objectif politique implicite présent dans tout discours historique. À cet égard, les techniques de manipulation par le langage sont particulièrement étudiées, surtout en classe de langues, lettres et de théorie de la connaissance. Sont particulièrement scrutées les particularités du langage politique, de la communication de guerre, du politiquement correct mais aussi le langage recourant à l'émotion, notamment en examinant des sources écrites ou des vidéos, comme par exemple le langage utilisé dans chaque camp politique pendant la guerre d'Irak à l'époque de George Bush ou, plus récemment, pendant la campagne présidentielle américaine.

La manipulation des visuels photographiques ou des vidéos est également étudiée, en recourant à des exemples précis, comme les photographies montrant Staline affichant une amitié pourtant non avérée avec Lénine, etc. Ces différentes pédagogies ont pour but de faire acquérir des réflexes de repérage de la falsification de documents, entre autres techniques de propagande.

Les élèves se montrent habiles à repérer ces procédés et à décrypter les matériaux de propagande et leurs messages idéologiques implicites, cette agilité s'affirmant bien sûr avec l'âge et la maturité. Cela les amène naturellement à opérer cette lecture critique dans leur usage quotidien des médias numériques. Certains deviennent capables de le faire de façon autonome.

Enfin, à la discrétion des professeurs, des cours sur les théories du complot peuvent être organisés en classe d'histoire ou de théorie de la connaissance. Plutôt que d'un cours, il s'agit d'abord de décrypter certaines théories du complot désormais " classiques", comme celles élaborées à propos de l'attentat du Wall Trade Center, via l'examen de différents matériels vidéo. Ensuite, il peut être demandé aux élèves, lors d'un exercice pratique, de se constituer en équipes et de fabriquer de toutes pièces une théorie du complot sur un thème choisi ou imposé. Une mini compétition en classe permet de féliciter et de primer l'équipe ayant fabriqué la " meilleure » théorie du complot. Cette créativité et ce recours à de multiples techniques vues et intégrées sont sans doute la meilleure confirmation de leur compréhension du phénomène.

\section{UN BRASSAGE CULTUREL PROPICE À L'EXAMEN DES VÉRITÉS HISTORIQUES}

La présence d'élèves ayant étudié dans d'autres pays fournit un avantage très spécifique à Singapour. Du fait de la présence de nationalités 
différentes au sein d'une même classe, beaucoup d'élèves ont étudié et suivi leur enseignement primaire voire le début du secondaire dans des pays de la région asiatique adoptant des programmes et vues historiques divergents. Ainsi les élèves ayant étudié au Japon, en Corée, en Birmanie, en Inde, en Chine, etc., abordent souvent les cours avec un bagage historique bien différent de celui des Singapouriens. Cette remarque vaut aussi pour les élèves nés à Singapour mais élevés dans les valeurs historiques et sociales d'une communauté ethnique particulière. Les vues, bien souvent hétérogènes et discordantes, concernant l'hégémonie politique américaine, le déclin du communisme, la défaite japonaise ou les crimes de guerre japonais, les guerres sino-japonaises ou encore la guerre de Corée, donnent lieu à des incompréhensions, à des réfutations à caractère parfois très émotionnel. Certains élèves peuvent se montrer offensés par les affirmations des élèves ou de l'enseignant, lorsqu'elles contredisent la "vérité historique " à laquelle ils étaient préalablement attachés. C'est bien souvent le cas des élèves chinois ayant appris à vénérer, dès les petites classes, toutes les figures historiques du communisme et qui souffrent de ne plus voir Lénine ou Mao Zedong jouir de leur statut de héros dans l'histoire enseignée à Singapour. L'enseignant tentera généralement de ne pas mettre l'élève dans une situation personnelle trop inconfortable. Ces remarques valent aussi pour les cours d'économie ou les théories économiques; le rôle des organisations économiques mondiales, les classements économiques mondiaux des pays ou encore l'éthique de l'économie sont des sujets qui donnent lieu à des controverses et exigent une flexibilité de la part des enseignants. Élèves comme enseignants se trouvent confrontés à des systèmes de valeurs et à des références historiques différents et contradictoires. Il devient alors difficile de proposer une approche homogène ou dogmatique. Même si les enseignements sont dominés par une vue occidentale du fait du choix des programmes (Cambridge et Baccalauréat international), dans les faits, les "vérités historiques " sont discutées en classe ; elles amènent l'enseignant à élargir ces problèmes spécifiques au problème général de la vérité historique et à endosser le rôle de l' « enseignant-citoyen du monde » expérimenté, à l'esprit ouvert plutôt que garant d'une vérité historique singulière, définie par des programmes scolaires particuliers.

En classe de théorie de la connaissance, les élèves vont plus loin dans cette approche critique de l'établissement de la vérité historique et sont invités à comparer la méthode scientifique aux méthodes utilisées en histoire pour l'établissement des théories reconnues et partagées collectivement. Cet exercice amène les élèves à réaliser la difficulté qui consiste à sélectionner les sources fiables, à rendre compte des biais que peuvent représenter les écoles de pensées auxquels appartiennent les journalistes, historiens ou auteurs de manuels scolaires. Les polémiques liées à la définition et à la sélection des contenus de manuels scolaires (au Japon ou au Texas par exemple) sont étudiées en classe et constituent une base d'exercice très vite assimilée par les élèves. 


\section{LA PENSÉE CRITIQUe ET LE RISQUE D'UNE ATTITUDE NIHILISTE}

Malgré toutes les singularités qui caractérisent l'élève à Singapour, celui-ci reste cependant, notamment en raison de son fort pouvoir d'achat, très exposé aux médias numériques. On regrettera que la quantité d'informations disponibles sur tout sujet et les apparentes contradictions entre elles produisent une forme de nihilisme chez les élèves. Ceux-ci finissent bien souvent par tomber dans le travers qui consiste soit à douter de tout et à ne plus croire en rien, soit à mettre toutes les informations au même rang d'égalité ou de valeur. Ces attitudes où «tout se vaut» sont particulièrement préjudiciables à la connaissance. Elles demandent un effort pédagogique aux enseignants, qui doivent repenser et renforcer les stratégies d'analyse critique de matériaux de toutes sortes.

Certains enseignants hésitent parfois à proposer des thèmes d'étude jusquelà considérés comme classiques, comme le négationnisme. $\mathrm{Si}$, a priori, l'analyse méthodique et scrupuleuse de documents semblait la bonne méthode, dans les faits et d'année en année, celle-ci montre ses limites. En analysant les textes des auteurs de déni (extraits de livres, discours, articles, interviews, etc.) les élèves finissent en effet par trouver ces documents plus convaincants que ceux justifiant l'existence avérée de l'Holocauste. Et pour cause, car pour gagner en crédibilité, les auteurs imitent bien souvent le style du discours scientifique ou documentaire. À titre d'exemple, des documents particulièrement détaillés démontrantsoi-disantl'impossibilité technique que le zyklon $\mathrm{B}$ ait pu être utilisé dans les chambres à gaz peuvent difficilement être critiqués par un professeur d'histoire qui ne serait pas en mesure de mettre le doigt sur les failles scientifiques de la démonstration. Il en va de même avec la fabrication de documentaires aux accents scientifiques, que l'on trouve à l'envi dans les théories du complot. Sur la forme, quelques outils méthodologiques sont transmis aux élèves pour aiguiser leur esprit critique : l'enseignant propose généralement des exercices pratiques, où il est demandé de procéder à l'examen scrupuleux des sources : la biographie de l'auteur et ses liens éventuels avec certaines idéologies, le lieu d'hébergement des sites pouvant indiquer une volonté d'échapper aux lois, mais aussi le style adopté (effets de montage, police de caractère, musique, commentaire narrativisé) et la rhétorique argumentative. Sur le fond, les approches face à ces sujets sensibles font parfois l'objet de travaux pluridisciplinaires. Des enseignants en langue, histoire et philosophie n'hésitent pas à collaborer, quitte à faire intervenir d'autres professeurs particulièrement légitimes pour aborder un sujet spécifique, afin de faire face à ces obstacles de la façon la plus convaincante possible. Malgré une évidente volonté de collaboration au service de la vérité, il faut faire ici l'aveu d'une certaine impuissance : le corps enseignant n'est, hélas, pas toujours capable de lutter contre la puissance du déferlement des informations non vérifiées ou fabriquées, favorisé par le phénomène des big data, qui abreuvent en un flux continu les cerveaux captifs des élèves, à Singapour comme ailleurs. 
Si le phénomène des big data n'est pas propre à Singapour, le ministère singapourien de l'éducation se montre cependant particulièrement sensible à cette question. Le projet de loi contre les fausses vérités et rumeurs va dans ce sens. Conscientes des effets secondaires que produit l'objectif d'excellence académique et qui se traduit parfois par l'apprentissage mécanique de leçons, des équipes pédagogiques réfléchissent à la manière de concilier performance académique et acquisition d'outils de réflexion. La compétence de pensée critique figure en effet parmi les compétences désirées chez tout élève ayant achevé le cycle secondaire. ${ }^{4}$ Ces dernières années, des programmes ont été mis en place pour éduquer les élèves à une pensée critique qui jusque-là manquait sans doute, ce qui n'est pas un hasard. Singapour, durant ses cinquante premières années d'existence, s'est construit sur l'effort remarquable de toute une population, qui, grâce à son travail, son assiduité et son courage, a su relever un défi économique que peu estimaient possible. Étant parvenue au niveau escompté, au plan local et international, Singapour peut maintenant affiner sa stratégie éducative et doter ses institutions scolaires et leurs élèves d'outils de réflexion plus élaborés. Un nouveau défi, en quelque sorte.

\section{BibliographIE}

DE KETELE J.-M. (2015) : "Les résultats des élèves asiatiques dans les enquêtes internationales", Revue internationale d'éducation de Sèvres, $\mathrm{n}^{\circ} 68$ [DOI : 10.4000/ ries.4351]

SEOW T., HO L. C. (2016) : «Singapore teachers' beliefs about the purpose of climate change education and student readiness to handle controversy", International Research in Geographical and Environmental Education, vol. 25, $\mathrm{n}^{\circ} 4$, p. 358-371 [DOI : 10.1080/10382046.2016.1207993]

TAN C. (2017) : " Teaching critical thinking: Cultural challenges and strategies in Singapore ", British Educational Research Journal, n 43, p. 988-1002 [DOI : 10.1002/ berj.3295]

4. Voir le site du ministère de l'éducation singapourien : [https://goo.gl/uWRS8G] 
